\title{
Brill Tagging on the Micron Automata Processor
}

\author{
Keira Zhou; Jeffrey J. Fox; Ke Wang; Donald E. Brown, Fellow IEEE; Kevin Skadron, Fellow IEEE \\ University of Virginia \\ Charlottesville, VA 22904 USA \\ \{qz4aq, jjf5x, kewang, brown, skadron\}@virginia.edu
}

\begin{abstract}
Semantic analysis often uses a pipeline of Natural Language Processing (NLP) tools such as part-of-speech (POS) tagging. Brill tagging is a classic rule-based algorithm for POS tagging within NLP. However, implementation of the tagger is inherently slow on conventional Von Neumann architectures. In this paper, we accelerate the second stage of Brill tagging on the Micron Automata Processor, a new computing architecture that can perform massive pattern matching in parallel. The designed structure is tested with a subset of the Brown Corpus using 218 contextual rules. The results show a $38 X$ speed-up for the second stage tagger implemented on a single AP chip, compared to a single thread implementation on CPU. This speed-up is linear with the number of rules, thus making large and/or complex rule sets computationally practical. This paper introduces the use of this new accelerator for computational linguistic tasks, particularly those that involve rule-based or pattern-matching approaches.
\end{abstract}

Keywords-Part-of-speech tagging; Brill tagging; Automata Processor; Natural Language Processing

\section{INTRODUCTION}

Semantic analysis often uses a pipeline of Natural Language Processing (NLP) tools such as part-of-speech (POS) tagging [14]. POS tagging makes assignments of a tag to input tokens, such as, nouns, verbs, adjectives, etc [1]. Accelerating these tools may be a promising approach for increasing the speed and potentially the sophistication of semantic analysis. We evaluate acceleration of POS tagging as a case study.

Brill Tagging is a classic rule-based POS tagging algorithm [9]. The algorithm is one of the most widely used rule-based approaches [6]. However, the computational time is slow for both training and tagging. Specifically, it may require RKn elementary steps to tag an input of $n$ words with $\mathrm{R}$ contextual rules with at most $\mathrm{K}$ tokens of context [8].

The Micron Automata Processor (AP) [10] is a novel non-Von Neumann architecture that can be programmed to run thousands of Non-deterministic Finite Automata (NFA), i.e. regular-expression rules, in parallel to identify patterns in a data stream. The work described here shows that the AP's parallelism can significantly

This work was supported by the Army Research Laboratory under grant number W911NF-10-2-0051; C-FAR, one of six centers of STARnet, a Semiconductor Research Corporation program sponsored by MARCO and DARPA; and a grant from Micron Technology. reduce the tagging time of Brill Tagging compared to implementation on a single-core CPU.

\section{BACKGROUND AND RELATED WORK}

\section{A. POS tagging}

Manually tagged corpora provide training data for automated taggers. POS tagging algorithms can be categorized into two groups: rule-based and stochastic (statistical) approaches. State-of-art stochastic based approaches include conditional random field models [7] and maximum entropy Markov models [12]. Brill tagging is one of the first and most widely used rule-based approaches [6]. When performing a POS tagging task, choosing a standard tagset is important. Two commonly used tagsets for POS tagging are the Brown (87 tags) [4] and Penn Treebank (36 tags) [5] tagsets.

The Brill algorithm proceeds in training and tagging steps. During the training, it identifies the most frequent tag for all recorded tokens as well as contextual rules for updating the tags. After training, a two-stage process tags new untagged corpora. The first stage assigns the most frequent tag to the new corpora. In the second stage, the initial tags are updated based on the contextual rules. Our work focuses on reducing the computational time of the second stage of the tagging by exploiting the fact that rules can be easily implemented in parallel as regular expressions on the AP.

\section{Automata PROCESSOR}

A physical embodiment of the AP is not yet available; however, we do have access to Micron's simulator (SDK) of the AP. This allows us to design automata and simulate the on-chip processes and performance.

There are three major components on the AP: StateTransition-Element (STE), Counter Element, and Boolean Elements, among which STE is the core component. The Counter and Boolean elements are not needed for Brill tagging. One STE can match an 8-bit user-specified symbol in a clock cycle and STEs can activate each other via a reconfigurable routing network. Each STE has three states: inactive, activated and matched. Only activated STEs will be able to inspect the next input symbol to perform a match against symbols

This is the authors' version of the final manuscript. The authoritative version can be found in the Proceedings of the 2015 IEEE International Conference on Semantic Computing, Feb. 2015. 
accepted by that STE. Once the symbol on an STE is matched, the STEs connected to it will be activated to accept the next input and match that against their programmed symbol matches. Table I provides illustrations of basic STE functions.

One AP chip contains a total of 49,152 STEs, among which 6144 can report. There are expected to be 32 chips on a PCI Express AP board, which thus has 1,572,864 STEs that can all operate in parallel [3]. The CPU initializes the AP chips, sends data, and fetches results using PCIe transactions. Given the AP chips' $7.5 \mathrm{~ns}$ clock period, one chip can process data at $128 \mathrm{M}$ symbols/sec; higher processing rates are possible by sharing the AP resources among multiple streams Our design easily fits inside a single AP chip, so multiple input streams or much larger rulesets could easily be supported.

\section{Design ON THE AUtomatA Processor}

\section{A. Brill Tagging initial steps}

Brill tagging is implemented in $\mathrm{C}$ and source code is openly available [2]. This implementation uses the Penn Treebank tagset and contains 218 contextual rules. We use this implementation to run the first stage tagging and write the intermediate result into a separate file that serves as the input for the second stage tagging.

\section{B. Design on the AP}

The input data for the AP consists of a stream of the words and initial tags. The rules are implemented on the AP as described below. All of the rules operate in parallel, inspecting the input stream for a potential match. Among the 218 rules, there are 19 different structures. The simplest rule structure matches a sequence of two tagged words, while the most complex structures look for patterns in sequences of up to seven tagged words. Fig. 1 provides an example design of an automaton with a simple structure that matches a sequence of two tags. We use " " to represent white space. The reporting element ID contains a rule ID as well as the update tag. This information is needed for post processing (Section IV.C.)

We illustrate the design with an example input:

$$
\text { ... to/TO conflict/NN with/IN... }
$$

The starting STE for the rule has a symbol "**". This means that the STE will be matched by any input symbols. The symbol on the second STE is "^/". Thus the second STE will be matched on any symbols that are NOT a "/". This activates both the third STE and itself. The self-activating function will keep the second STE activated and accepting input symbols until a "/" appears. This ignores the actual words; the Brill rules are based on patterns of tags. When the "/" is seen, the third STE will be matched and activate the forth STE; the next characters, up to a " $"$ ", constitute a tag.
TABLE I. GRAPHIC ILLUSTRATIONS OF BASIC STE FUNCTIONS

\begin{tabular}{|c|c|}
\hline & $\begin{array}{l}\text { A starting STE: It can either be start-of-data, which } \\
\text { only matches the first symbol of the input and matches } \\
\text { against A; or all-input-start, which accepts every } \\
\text { symbol from the input and matches against Symbol A }\end{array}$ \\
\hline & $\begin{array}{l}\text { Matching - Activating: Symbol A is matched on the } \\
\text { first STE, and the second STE is activated; a "*" means } \\
\text { to match any input symbols; " " " means a negation of } \\
\text { the symbol }\end{array}$ \\
\hline & $\begin{array}{l}\text { Self-activating: The STE activates itself when Symbol } \\
\text { A is matched }\end{array}$ \\
\hline & $\begin{array}{l}\text { A reporting STE: Reports when the symbol A is } \\
\text { matched }\end{array}$ \\
\hline
\end{tabular}

This design assumes that every word is followed by a "/" and then its tag. Each word/tag pair ends with a " ". If, at any point, a rule no longer matches the input, the “*” STE keeps the rule actively processing subsequent input.

\section{Post processing}

As the input data stream is being inspected by the rules on the AP, reporting elements generate output whenever a match is found. The output consists of an offset number that specifies the input symbol cycle on which one or more reporting elements fired, as well as the ID of the reporting elements. A post processing step is needed to use this output to update the tags and thus complete the second stage of Brill Tagging. We modified the openly available Brill code to conduct the post processing. In the original code, a word and a tag array are created when reading in the first-stage-tagged file. Our post processing requires another array that matches each character in the file with the word to which the character belongs. Table II shows this array for the example sentence we used previously.

After all the arrays are created, the baseline CPU code then reads in the contextual rule file that contains 218 rules and applies one rule at a time to the entire corpus. For the AP, we modified this part of the code. Instead of reading in the contextual rule file, the code now reads the output file from the AP Emulator and performs postprocessing. Both rule ID and the update tag information can be obtained from the ID of the reporting STEs. The offset number indicates the character position at which an entire rule is matched. Using the offset number, we can use the character position array to look up the index of the word that needs to be updated. An example report we get from the AP looks like:

Offset $28 \quad$ Reporting Element ID: rule2_VB 


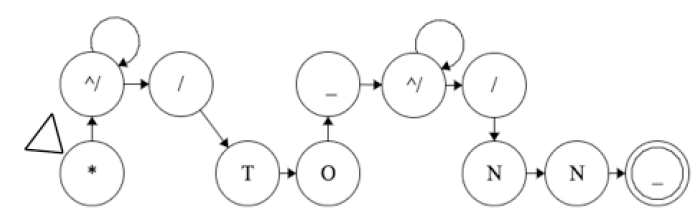

Figure 1. Example design of a simple automaton structure for the rule described in Section IV.B.

From the character position array, we find that the word associated with the $28^{\text {th }}$ character is the $6^{\text {th }}$ word. We thus update the tag of that word in the original corpus.

Fig. 2 shows the steps involved for the CPU and AP implementation. The bold-italicized parts are the execution time we included in the comparison. For the original Brill code on the CPU, for the most direct comparison with the AP, we only count the step for reading the rules, matching the rules, and updating tags. For the AP implementation, we include the estimate of the matching time on the AP (see Fig. 3, last column), and the pre/post processing time on the CPU to: 1) create the character position array; 2) read the file with the reported rules and 3) update the tags. The steps we ignore are identical in both the CPU and AP implementations.

\section{Test Data And Result}

\section{A. Test data}

To test our design we use a subset of the Brown Corpus [4] downloaded from the NLTK website [7]. We combined the files into 5 different sizes: $40 \mathrm{~KB}, 60 \mathrm{~KB}$, $79 \mathrm{~KB}, 99 \mathrm{~KB}$ to test the impact of input size on execution time for both the CPU and AP implementations. We also tested the largest file $(99 \mathrm{~KB})$ with different numbers of rules ranging from 20 to 218 rules to test the impact of the number of rules on the execution time.

TABLE II. The CHARACTER POSITION ARRAY

\begin{tabular}{|l|c|c|c|c|c|c|c|}
\hline Characters & $\ldots$ & t & o & $/$ & T & O & - \\
\hline $\begin{array}{l}\text { Index of the Char } \\
\text { Position Array }\end{array}$ & $\ldots$ & 11 & 12 & 13 & 14 & 15 & 16 \\
\hline $\begin{array}{l}\text { Array Content (Index } \\
\text { of Word Array) }\end{array}$ & $\ldots$ & 4 & 4 & 4 & 5 & 5 & 5 \\
\hline \hline Characters & $\mathbf{c}$ & $\mathbf{o}$ & $\mathbf{n}$ & $\mathbf{f}$ & $\mathbf{I}$ & $\mathbf{i}$ & $\mathbf{c}$ \\
\hline Index & 17 & 18 & 19 & 20 & 21 & 22 & 23 \\
\hline Array Content & 5 & 5 & 5 & 5 & 5 & 5 & 5 \\
\hline \hline Characters & $\mathbf{t}$ & $/$ & $\mathbf{N}$ & $\mathbf{N}$ & - & $\mathbf{w}$ & $\mathbf{i}$ \\
\hline Index & 24 & 25 & 26 & 27 & 28 & 29 & 30 \\
\hline Array Content & 5 & 5 & 5 & 5 & 5 & 6 & 6 \\
\hline \hline Characters & $\mathbf{t}$ & $\mathbf{h}$ & $/$ & $\mathbf{I}$ & $\mathbf{N}$ & - & $\ldots$ \\
\hline Index & 31 & 32 & 33 & 34 & 35 & 36 & $\ldots$ \\
\hline Array Content & 6 & 6 & 6 & 6 & 6 & 6 & $\ldots$ \\
\hline
\end{tabular}

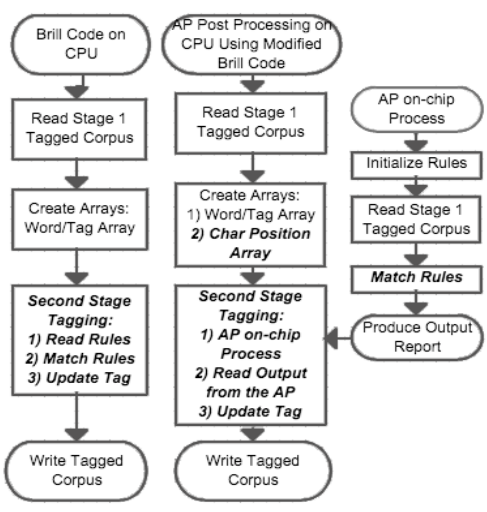

Figure 2. The CPU and AP Processes

\section{B. Execution environment}

For the CPU implementation, we used the published $\mathrm{C}$ code [2] and ran it on an Intel Core i5 machine with a single thread. The execution time recorded for the CPU implementation is the wall clock time for the step of updating the tags measured in microseconds.

For the AP implementation, one character is processed per clock cycle, so on-chip time is a direct function of the input size. Post processing is conducted on the same CPU. The execution time included for the post processing is the wall clock time for the steps of creating the extra character position array and updating the tags based on the output report from the AP.

\section{Results}

\section{1) Execution time for different input data size}

Table IV shows the execution time of the CPU and AP implementation for different sizes of input data (Time in microsecond). The speed-ups are within the range of $38.3 \mathrm{X}$ to $41.0 \mathrm{X}$. This suggests that the size of the input does not have a significant impact on the speed-up.

\section{2) Execution time for different number of rules}

Fig. 3 shows the speed-up in relation to number of rules. We can see that there is an approximately linear growth of the speed-up with the number of rules. Although we only tested with up to 218 rules, the largest number of rules mentioned in the literature is 1729 [12]. Based on the regression line $(\mathrm{y}=0.1577 \mathrm{x}+3.3794)$, we estimate that the potential speed-up for such a rule set could be $276.0 \mathrm{X}$.

TABLE III. EXECUTION TIME FOR DIFFERENT SIZES OF INPUT

\begin{tabular}{|c|c|c|c|c|c|}
\hline \multicolumn{2}{|c|}{$\begin{array}{c}\text { Time in } \\
\text { microsecond }\end{array}$} & $40 \mathrm{~KB}$ & $60 \mathrm{~KB}$ & $79 \mathrm{~KB}$ & $99 \mathrm{~KB}$ \\
\hline \multicolumn{2}{|r|}{ CPU } & 56130 & 86545 & 112289 & 141810 \\
\hline \multirow{4}{*}{$\begin{array}{l}\mathbf{A} \\
\mathbf{P}\end{array}$} & On chip & 298 & 453 & 594 & 741 \\
\hline & Create Array & 372 & 596 & 875 & 1031 \\
\hline & Post process & 747 & 1063 & 1462 & 1935 \\
\hline & Total & 1417 & 2112 & 2931 & 3707 \\
\hline \multicolumn{2}{|r|}{ Speed-up } & $39.6 \mathrm{X}$ & $41.0 \mathrm{X}$ & $38.3 \mathrm{X}$ & $38.3 \mathrm{X}$ \\
\hline
\end{tabular}




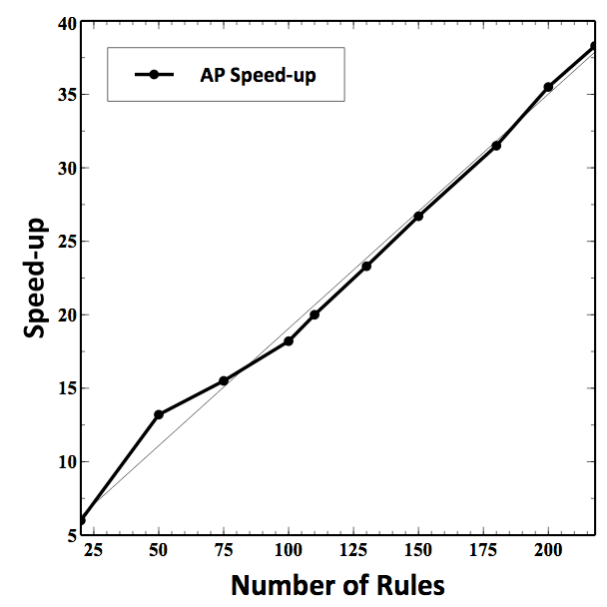

Figure 3. Speedup in Relation to Number of Rules

\section{ACCURACY AND DISCREPANCY}

The Association for Computational Linguistics provides the accuracies of several POS tagging systems for the Wall Street Journal corpus [13]. The accuracy of Brill Tagging is listed at $96.5 \%$, while Hidden Markov model is $96.96 \%$ and Maximum Entropy Markov Model is $97.32 \%$. The implementation of Brill Tagging described here could make larger and more complex rule sets computationally practical. Such rule sets could allow Brill Tagging to achieve higher accuracy. On the other hand, the CPU implementation of Brill Tagging applies one rule at a time, while the AP implementation matches all rules in parallel, which can lead to differences in updating tags. For example, in the CPU version, for a given word, after rule 1 is applied and the tag is updated, rule 2 may not be triggered; while in the AP version, both rules will be triggered, thus the order of updating the tag will depend on the AP output. We used 4 different input files to estimate these discrepancies. We obtained the annotated Brown Corpus, which used the Brown tagset to determine the correct tag when the two methods disagreed. However, the 218 rules within the published software use the Penn Treebank tagset. Thus, the correct tags for a few tag differences were categorized as unknown. To set an upper bound for the decrease in accuracy, we count all unknown differences for the CPU implementation as correct and AP implementation as wrong. Table IV shows that such discrepancies are rare.

\section{TABLE IV. TAGGING DISCREPANCIES FOR DIFFERENT SAMPLES}

\begin{tabular}{|c|c|c|c|c|}
\hline & $\begin{array}{c}\text { ca01 } \\
\mathbf{( 2 2 4 2} \\
\text { words) }\end{array}$ & $\begin{array}{c}\text { cb01 } \\
\mathbf{( 2 2 0 0} \\
\text { words) }\end{array}$ & $\begin{array}{c}\text { cc01 } \\
\mathbf{( 2 4 1 5} \\
\text { words) }\end{array}$ & $\begin{array}{c}\text { cd01 } \\
\mathbf{( 2 2 1 3} \\
\text { words) }\end{array}$ \\
\hline CPU Correct & 5 & 2 & 6 & 6 \\
\hline AP Correct & 2 & 1 & 1 & 3 \\
\hline Both Wrong & 0 & 1 & 1 & 1 \\
\hline Unknown & 2 & 3 & 2 & 5 \\
\hline $\begin{array}{c}\text { Difference in } \\
\text { Accuracy }\end{array}$ & $0.223 \%$ & $0.182 \%$ & $0.290 \%$ & $0.362 \%$ \\
\hline Average & \multicolumn{5}{|c|}{$0.264 \%$} \\
\hline
\end{tabular}

\section{CONCLUSIONS AND FUTURE WORK}

The Micron AP is a novel non-Von Neumann architecture that can test for thousands or even hundreds of thousands of patterns in parallel. This paper introduces an AP implementation of Brill tagging, and shows that the AP achieves a significant speed-up.

We plan to implement this design on hardware once the AP is available. In addition, we will more rigorously evaluate the accuracy of the CPU and AP designs relative to each other (combining rules may be one promising approach to reduce differences in tagging) and relative to state-of-the-art stochastic POS-tagging techniques.

This study suggests that the AP may be a promising platform for other semantic analysis tasks. We plan to explore other applications within NLP domain such as parsing and machine translation.

\section{ACKNOWLEDGMENTS}

The authors thank Mateja Putic for assistance in the initial setup, Micron Technology for providing access to the AP SDK, Matt Tanner for his insights into using the $\mathrm{AP}$, and the anonymous reviewers for their helpful comments.

\section{REFERENCES}

[1] Voutilainen, A. "Part-of-speech tagging." The Oxford handbook of computational linguistics (2003): 219-232.

[2] Brill, E. CIS, Univ. of Pennsylvania, and the Spoken Language Systems Group, Laboratory for Computer Science, MIT, 1994.

http://www.tech.plym.ac.uk/soc/staff/guidbugm/software/RULE_ BASED_TAGGER_V.1.14.tar.Z

[3] Roy, I. and S. Aluru. "Finding Motifs in Biological Sequences Using the Micron Automata Processor." IPDPS, 2014.

[4] Francis, W.N. and H. Kucera. Brown Corpus Manual, 1964.

[5] Santorini, B. "Part-of-speech tagging guidelines for the Penn Treebank Project (3rd revision)." 1990.

[6] Mohammad, S. and T.Pedersen. "Guaranteed pre-tagging for the brill tagger." CICLing'03. Springer Berlin, 2003. 148-157.

[7] NLTK Corpora. Natural Language Toolkit. Web. 2013.

[8] Roche, E. and Y. Schabes. "Deterministic part-of-speech tagging with finite-state transducers." Computational linguistics 21.2 (1995): 227-253.

[9] Brill, E. "Transformation-based error-driven learning and natural language processing: A case study in part-of-speech tagging." Computational linguistics21.4 (1995): 543-565.

[10] Dlugosch, P., D. Brown, P. Glendenning, M. Leventhal, and H. Noyes, "An efficient and scalable semiconductor architecture for parallel automata processing," IEEE TPDS, 2014.

[11] Brill, E. "Unsupervised learning of disambiguation rules for part of speech tagging." WVLC-3. Vol. 30. New Jersey: ACL, 1995.

[12] McCallum, A., D. Freitag, and F. CN Pereira. "Maximum Entropy Markov Models for Information Extraction and Segmentation." ICML. 2000.

[13] "POS Tagging (State of the art)". Wiki of the ACL. Web. 2013.

[14] Sheu, P., et al., eds. Semantic Computing. John Wiley \& Sons, 2011. 